\title{
Chromosome Identification and Karyotyping of Satsuma Mandarin by Genomic In Situ Hybridization
}

\author{
Akira Kitajima ${ }^{1}$, Atsu Yamasaki, and Tsuyoshi Habu \\ Experimental Farm, Graduate School of Agriculture, Kyoto University, Takatsuki City, Osaka \\ 569-0096, Japan
}

\author{
Bannarat Preedasuttijit and Kojiro Hasegawa \\ Faculty of Agriculture, Kochi University, Nankoku City, Kochi 783-0093, Japan
}

\begin{abstract}
Additional INDEX words. Citrus unshiu, CMA/DAPI, genomic similarity, GISH, karyotype evolution, pummelo
Aвstract. Satsuma mandarin (Citrus unshiu Marcow.) chromosomes were stained with Giemsa and fluorochromes chromomycin $\mathrm{A}_{3}$ (CMA)/4',6-diamidino-2-phenyindole (DAPI). Eighteen chromosomes were categorized into eight groups by the position and relative size of the CMA $(+)$ region and relative length of chromosome. Ponkan $(C$. reticulata Blanco) DNA labeled with Dig-rhodamine (red) and pummelo [C. maxima (Burm.) Merr.] DNA labeled with biotin-fluorescein isothiocyanate (green) were used as genomic in situ hybridization (GISH) probes. GISH signals were detected on CMA $(+)$ regions and other heterochromatin blocks. The chromosomes were categorized into 12 groups by the coloration and size of GISH signals with relative length of chromosomes. GISH allowed six pairs of speculated homozygous and six individual heterozygous chromosomes of satsuma mandarin to be identified unambiguously. In 10 chromosomes with distinct GISH signals on the CMA $(+)$ regions, red GISH signals were detected on nine chromosomes, indicating that satsuma mandarin is closely related to ponkan. Two colors (red and green) of GISH signals were detected on type $\mathrm{C}$ chromosome and three different colors (red, green, and yellow) were detected on type $\mathrm{A}$, indicating that pummelo is involved in the origin of satsuma mandarin. The origins of types $\mathrm{A}$ and $\mathrm{C}$ chromosomes in satsuma mandarin were also discussed. This article demonstrates that GISH is a powerful tool for chromosome identification and karyotyping in citrus.
\end{abstract}

\begin{abstract}
Satsuma mandarin, the most important citrus in Japan, initiate as a chance seedling in Japan (Nagashima Island, Kagoshima Prefecture), but its origin is unclear. Satsuma mandarin has many horticultural quality advantages, including juiciness, sweetness, low acidity, seedlessness, and easy peeling. It also features cultivation advantages as disease tolerance (Yoshida and Mitsuoka, 1993), cold hardiness (Davies and Albrigo, 1994b; Yoshimura et al., 1963), parthenocarpy (Ueno and Shichijo, 1976; Yamamoto et al., 1995), and male sterility (Nakano et al., 2001). The construction of linkage maps and the mapping of useful inheritance genes for breeding have recently been developed in citrus using molecular tools (Cai et al., 1994; Garcia et al., 1999; Ruiz and Asins, 2003; Sankar and Moore, 2001). It is important to confirm the correlation of linkage group with each chromosome. For chromosome mapping, the identification of each chromosome and detailed karyotyping techniques must be established.
\end{abstract}

Although the citrus chromosome number is relatively small $(2 n=18)$, karyotyping is difficult because metaphase chromosomes are very small and morphologically similar. Fluorochromes chromomycin A3 staining is useful for identification of citrus chromosome types (Befu et al., 2000; Carvalho et al., 2005; Guerra, 1993; Miranda et al., 1997a; Yamamoto and Tominaga, 2003). Miranda et al. (1997a) and Befu et al. (2000) classified citrus chromosomes in five types (A to E) depending on the patterns of chromomycin $\mathrm{A}_{3}(\mathrm{CMA})$-positive [CMA (+)] regions (Fig. 1). Type A chromosome has three CMA (+)

Received for publication 11 Jan. 2007. Accepted for publication 4 Aug. 2007. ${ }^{1}$ Corresponding author. E-mail: kitajima@kais.kyoto-u.ac.jp. regions in the terminals of both arms and a proximal; type $\mathrm{B}$ has two CMA (+) regions in a terminal of one arm and a proximal; type $\mathrm{C}$ has two CMA $(+)$ regions in terminals of both arms; type D has one CMA (+) region in a terminal of one arm; and type $\mathrm{E}$ has no CMA (+) region. Furthermore, Befu et al. (2001) reported that the karyotype of satsuma mandarin was $1 \mathrm{~A}+1 \mathrm{C}+8 \mathrm{D}+8 \mathrm{E}$ in addition to two longer and two shorter chromosomes of type E. However, chromosome identification among type $\mathrm{D}$ chromosomes and the remaining type $\mathrm{E}$ chromosomes was difficult.

Karyological analysis for evolutionary relationships has been performed by heteromorphic chromosome pair comparisons based on CMA banding patterns and rDNA sites in the mandarins (Cornelio et al., 2003) and the lemon-lime group (Carvalho et al., 2005). Although these approaches attained some progress regarding citrus evolutionary relationships, new information is necessary for more detailed karyological analysis.

Genomic in situ hybridization (GISH) has become a useful tool for the characterization of genomes and chromosomes in polyploids and somatic hybrids of herbaceous plants (Raina and Rani, 2003), and GISH for identification of parental chromosomes in somatic hybrids of fruit trees were also reported in Diospyros kaki L. + D. glandulosa Lace. (Choi et al., 2002) and in Citrus aurantium L. + Poncirus trifoliata (L.) Raf. (Fu et al., 2004). Double-target GISH in which the total genomic DNA of two species is used as probes, has been effective for the identification of individual chromosomes of nonsomatic hybrids plants in Brassica L. species [B. nigra (L.) Koch, $B$. campestris L., and B. juncea (L.) Czerniak et Coss] (Maluszynska and Hasterok, 2005). Moreover, GISH was 
0

A

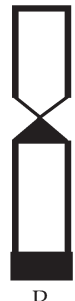

$\mathrm{B}$

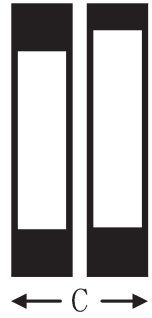

$\mathrm{C}_{1} \mathrm{C}_{2}$

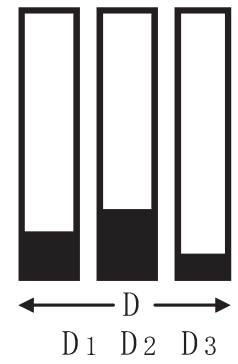

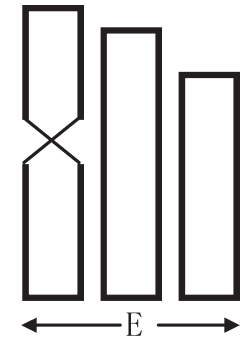

EL EM ES
Fig. 1. Schematic representation of chromosome types in citrus depend on the patterns of chromomycin $\mathrm{A}_{3}$ (CMA)-positive $(+)$ regions (filled with black). The type A chromosome has three CMA $(+)$ regions in terminals of both arms and a proximal, type $\mathrm{B}$ has two CMA $(+)$ regions in a terminal of one arm and a proximal, type $\mathrm{C}$ has two CMA $(+)$ regions in terminals of both arms, type $\mathrm{D}$ has one CMA $(+)$ region in a terminal of one arm, and type E has no CMA $(+)$ region. Detail classification of the type $\mathrm{C}, \mathrm{D}$, and $\mathrm{E}$ chromosomes with the relative sizes of CMA $(+)$ regions. The $\mathrm{C} 1$ has two $\mathrm{CMA}(+)$ regions with almost the same size and $\mathrm{C}_{2}$ has two CMA $(+)$ regions with obviously different sizes. The $\mathrm{D}_{1}, \mathrm{D}_{2}$, and $\mathrm{D}_{3}$ has a CMA $(+)$ region with medium size, relatively large, and relatively small, respectively. The relative chromosome length in $\mathrm{E}_{\mathrm{M}}, \mathrm{E}_{\mathrm{L}}$, and $\mathrm{E}_{\mathrm{S}}$ is medium, longer than $\mathrm{E}_{\mathrm{M}}$, and shorter than $\mathrm{E}_{\mathrm{M}}$, respectively.

useful to analyze evolutionary relationships among Zea mays $\mathrm{L}$. subspecies (Z. mays ssp. mays, Z. mays ssp. parviglumis Iltis et Doebley, and $Z$. mays ssp. huehuetenanguensis Doebley) (Poggio et al., 2005). The objectives of the present study were to identify individual chromosomes and analyze chromosome evolution in satsuma mandarin by double-target GISH.

\section{Materials and Methods}

Chromosome preparation. The method for chromosomespread preparation followed that of Kitajima et al. (2001). Young leaves ( 3 to $5 \mathrm{~mm}$ long) of 'Nankan No. 20' satsuma mandarin (C. unshiu) were treated with $2 \mathrm{~mm}$ 8-hydroxyquinoline (Nakalai Tesque, Kyoto, Japan) for $3 \mathrm{~h}$ at $20{ }^{\circ} \mathrm{C}$, fixed in methanol:acetic acid (3:1), and stored at $5{ }^{\circ} \mathrm{C}$ until used. Fixed leaves were washed in distilled water, cut finely, and then digested in $0.3 \%$ Cellulase Onozuka RS (Yakult Co. Ltd., Tokyo) and $0.2 \%$ Pectolyase Y-23 (Kikkoman Corp., Tokyo) in hypotonic solution $\left(75 \mathrm{~mm} \mathrm{KCl}\right.$ plus $7.5 \mathrm{~mm} \mathrm{Na} \mathrm{Na}_{2}$-EDTA, $\mathrm{pH} 4.0$ ) for $3 \mathrm{~h}$ at $37^{\circ} \mathrm{C}$. After digestion, the solutions of the macerated cells were mixed gently, added to the hypotonic solution, and centrifuged at $200 g_{\mathrm{n}}$ for $5 \mathrm{~min}$. The supernatant was removed and washed in a fixative solution. The macerated cells were resuspended in a small amount of fixative solution and dropped onto a glass slide. The chromosome preparations were stained with a $2 \%$ Giemsa solution (Sigma-Aldrich Co., St. Louis, MO), and the spread quality was checked with a microscope (BX-50; Olympus Corp., Tokyo). Good spread chromosomes were photographed with a microscope camera system (PM-20; Olympus + Fuji color film ISO 100; Fujifilm Corp., Tokyo). Satisfactory preparations were destained by washing with $70 \%$ ethanol and air-dried for CMA staining.

CMA/4, 6-diamidino-2-Phenyindole staining. The CMA staining procedure followed that of Befu et al. (2000). Preparations were sequentially treated with McIlvaine's buffer, 0.2 $\mathrm{mg} \cdot \mathrm{mL}^{-1}$ distamaycin A (Sigma-Aldrich Co.), a $5.0 \mathrm{~mm} \mathrm{MgCl}_{2}$ buffer solution, $0.12 \mathrm{mg} \cdot \mathrm{mL}^{-1} \mathrm{CMA}$ (Sigma-Aldrich Co.), and then rinsed with Mcllvaine's buffer. They were counterstained with 4, 6-diamidino-2-phenyindole (DAPI) (Sigma-Aldrich Co.) and mounted in a $p$-phenylene diamine (PPD) antifade solution. Chromosomes of CMA and DAPI staining were photographed with an epifluorescence microscope (BX-FLA; Olympus Corp.) and a camera system (PM-20 + Fuji color film ISO 100) equipped with BV (475 nm) and WU (420 nm) filter, respectively. Preparations were destained by washing with ethanol:acetic acid $(3: 1)$ and dehydration in a $70 \%-90 \%-$ $100 \%$ ethanol series for GISH.

Genomic IN SITU hYBRidization. The total genomic DNA of 'Yoshida' ponkan (C. reticulata) and 'Banpeiyu' pummelo (C. maxima) were extracted from immature leaves by a CTAB method, and the DNA concentration was adjusted to $100 \mathrm{ng} \cdot \mu \mathrm{L}^{-1}$. The total genomic DNA of the ponkan was labeled with digoxigenin-11-dUTP (Dig) and the pummelo DNA was labeled with biotin-16-dUTP (Bio) using a nick translation kit according to the manufacturer's protocol (La Roche Ltd., Basel, Switzerland). Each labeled DNA was resolved in $20 \mu \mathrm{L}$ formamide (probe solution), and the solution was mixed in $5 \mu \mathrm{L}$ of the Dig-labeled probe solution and in $10 \mu \mathrm{L}$ of the Bio-labeled probe. The probe mixture was denatured at $80{ }^{\circ} \mathrm{C}$ for $10 \mathrm{~min}$ and then added to $15 \mu \mathrm{L}$ of $20 \%$ dextran sulfate (Sigma-Aldrich Co.) in $4 \times$ SSC.

After aging the preparation in $4 \times \mathrm{SSC}$ with a $0.1 \%$ Triton $\mathrm{X}$ (Sigma-Aldrich Co.) at $37{ }^{\circ} \mathrm{C}$ for $30 \mathrm{~min}$ and dehydration, chromosome DNA of the preparation was denatured in $2 \times \mathrm{SSC}$ with $70 \%$ formamide at $70{ }^{\circ} \mathrm{C}$ for $5 \mathrm{~min}$. Denatured probe mixture was dropped onto the preparation and covered with parafilm and then hybridized overnight in a humid chamber at $37^{\circ} \mathrm{C}$. After washing with $50 \%$ formamide in $2 \times \mathrm{SSC}$ at $37^{\circ} \mathrm{C}$ for $15 \mathrm{~min}$ and $2 \times \mathrm{SSC}$ and $1 \times \mathrm{SSC}$ for $15 \mathrm{~min}$, the immunodetections of Dig-labeled and Bio-labeled DNAs were carried out with rhodamine-conjugated anti-Dig and fluorescein isothiocyanate-conjugated avidin (La Roche Ltd.), respectively. Preparations were counterstained with DAPI and mounted in PPD. Chromosomes of GISH were photographed with an epifluorescence microscope (BX-FLA) and a camera system (PM-20, Fuji color film ISO 100) by double exposure equipped with BV and IG (575 to $625 \mathrm{~nm}$ ) filters.

\section{Results and Discussion}

Giemsa and Chromomycin A $\mathbf{3}$ /DAPI staining. Chromosomes of 'Nankan No. 20' satsuma mandarin stained with Giemsa, DAPI, and CMA are shown in Figures $2 \mathrm{~A}-\mathrm{C}$, respectively. The total numbers of CMA $(+)$ signals were 13 in 18 chromosomes. CMA $(+)$ regions of satsuma mandarin were DAPI $( \pm)$ or DAPI $(+)$, whereas $\mathrm{CMA}(+) / \mathrm{DAPI}(-)$ and CMA(+)/DAPI(-) regions were reported for citrus (Carvalho et al., 2005; Cornelio et al., 2003; Guerra, 1993; Matsuyama et al., 1996; Miranda et al., 1997a; Pedrosa et al., 2000). The karyotype of 'Nankan No. 20' satsuma mandarin was $1 \mathrm{~A}+1 \mathrm{C}+8 \mathrm{D}+8 \mathrm{E}$ based on the patterns of the CMA $(+)$ regions (Figs. 2C and 3). This result agrees with the karyotype of 'Okitsu Wase' satsuma mandarin reported by Befu et al. (2001). They also reported that two longer and two shorter type E chromosomes were distinguishable in 'Okitsu Wase' satsuma mandarin. In this study, two longer and two shorter type E chromosomes were also distinguishable in 'Nankan No. 20' satsuma mandarin. In addition, the centromere region was recognized in two longer type $\mathrm{E}$ chromosomes.

Befu et al. (2002) reported that chromosomes with the same types of CMA-banding patterns were classifiable on the basis of relative sizes in the CMA $(+)$ regions. Following the manner of 

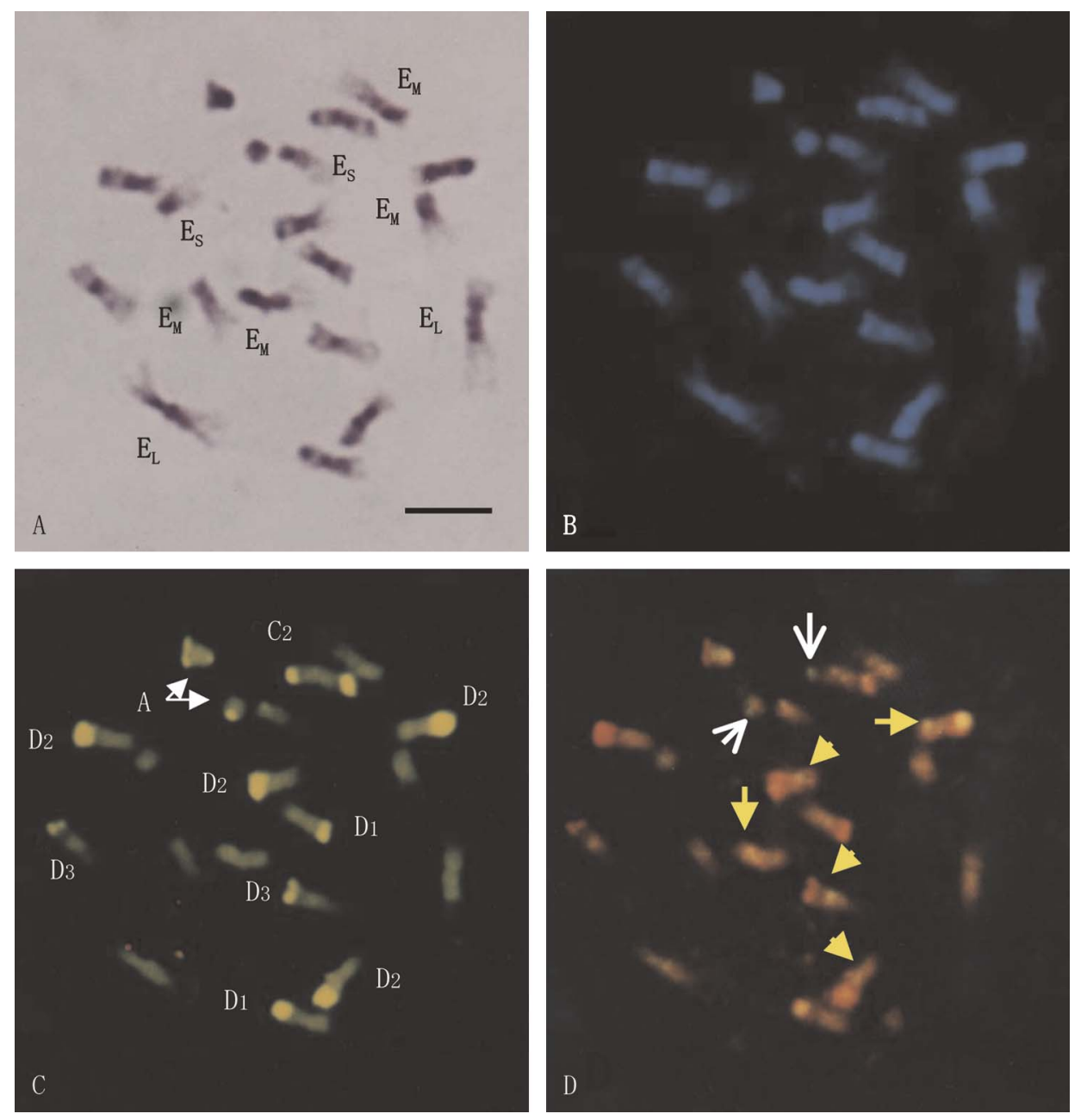

Fig. 2. Photographs of satsuma mandarin chromosome. (A) Giemsa staining, type E chromosomes [no chromomycin $\mathrm{A}_{3}$ (CMA)-positive (+) regions] of $\mathrm{E}_{\mathrm{L}}$ (long in chromosome length), $\mathrm{E}_{\mathrm{M}}$ (medium), and $\mathrm{E}_{\mathrm{S}}$ (short). Bar indicates $5 \mu \mathrm{m}$. (B) 4, 6-diamidino-2-phenyindole (DAPI) staining. (C) CMA staining, type A chromosome [three CMA $(+)$ regions], type $\mathrm{C}_{2}$ [different sizes of two CMA $(+)$ regions], and type $\mathrm{D}$ chromosomes [one CMA $(+)$ region] of $\mathrm{D}_{1}$ [medium size CMA $(+)$ region], $\mathrm{D}_{2}$ [large CMA $(+)$ region], and $\mathrm{D}_{3}$ [small CMA (+) region]. (D) Genomic in situ hybridization (GISH) using double-probe DNA from 'Yoshida' ponkan detected with red rhodamine and from 'Banpeiyu' pummelo detected with green fluorescein isothiocyanate (FITC); white arrows $(\rightarrow$ ) indicate green signals and yellow arrows $(\rightarrow)$ indicate yellow signals detected on no CMA $(+)$ regions.

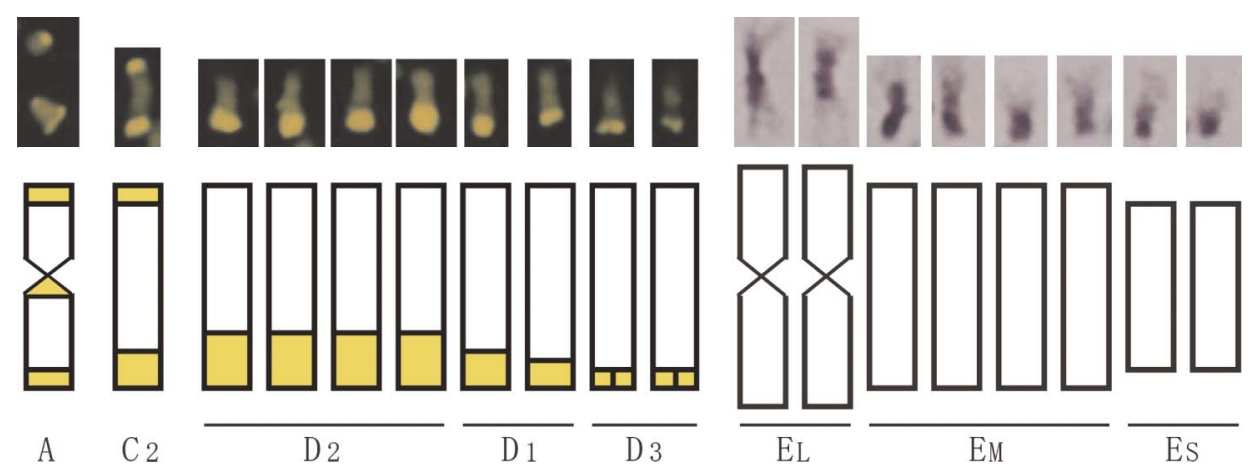

Fig. 3. Idiogram with picture images of satsuma mandarin chromosomes by chromomycin $\mathrm{A}_{3}(\mathrm{CMA})$ and Giemsa staining. Chromosomes were grouped into eight categories by the position and relative size of the CMA-positive $(+)$ regions (see Figs. 1 and $2 \mathrm{C}$ ) and relative length of the type E chromosomes (see Figs. 1 and $2 \mathrm{~A}$ ). The karyotype is $1 \mathrm{~A}+1 \mathrm{C}_{2}+2 \mathrm{D}_{1}+4 \mathrm{D}_{2}+2 \mathrm{D}_{3}+2 \mathrm{E}_{\mathrm{L}}+4 \mathrm{E}_{\mathrm{M}}+2 \mathrm{E}_{\mathrm{S}}$.

Befu et al. (2002) with minor modifications, as shown in Figure 1, we classified the chromosomes of types $\mathrm{C}$ to $\mathrm{C}_{1}$ [two CMA (+) regions, almost the same size], $\mathrm{C}_{2}$ [two CMA (+) regions, obviously different sizes], and the chromosomes of types $\mathrm{D}$ to $\mathrm{D}_{1}$ [medium size, CMA $(+)$ regions], $\mathrm{D}_{2}$ [relatively large, CMA (+) region], and $\mathrm{D}_{3}$ [relatively small, CMA $(+)$ region]. Moreover, the chromosomes of type $\mathrm{E}$ were classified on the basis of relative chromosome length to $\mathrm{E}_{\mathrm{M}}$ (medium length), $\mathrm{E}_{\mathrm{L}}$ (relatively longer than $E_{M}$ ), and $E_{S}$ (relatively shorter than $\left.E_{M}\right)$. The results in this study show that one type $\mathrm{C}$ chromosome was classified to $\mathrm{C}_{2}$ (Figs. $2 \mathrm{C}$ and 3). Of eight chromosomes of type $\mathrm{D}$, four chromosomes were $\mathrm{D}_{2}$, two were $\mathrm{D}_{1}$, and two were $\mathrm{D}_{3}$. Of eight chromosomes of type $\mathrm{E}$, two chromosomes were $E_{L}$, four were $E_{M}$, and two were $\mathrm{E}_{\mathrm{S}}$. From the results, 18 chromosomes of 'Nankan No. 20' satsuma mandarin were categorized into eight groups and the karyotype was confirmed to be $1 \mathrm{~A}+1 \mathrm{C}_{2}+2 \mathrm{D}_{1}+4 \mathrm{D}_{2}+$ $2 \mathrm{D}_{3}+2 \mathrm{E}_{\mathrm{L}}+4 \mathrm{E}_{\mathrm{M}}+2 \mathrm{E}_{\mathrm{S}}$ by Giemsa and CMA staining (Fig. 3).

Chromosome IDENTIFICATION BY GENOMIC IN SITU HYBRIDIZATION. There were more than 13 distinct GISH signals located on at least each CMA (+) region (Fig. 2C-D). That is, GISH signals were detected in all CMA (+) regions. CMA has a higher affinity for GC-rich DNA and DAPI for AT-rich DNA. However, CMA (+) regions were not DAPI (-) or DAPI $(-)$ (Fig. 2B-C). On the other hand, we confirmed that PI $(+)$ regions were identical to CMA $(+)$ regions in citrus chromosomes (Yamaguchi et al., 2002). PI has no specific affinity for any DNA bases. These facts indicate that many CMA $(+)$ regions in citrus are not relatively GC-rich for AT, although some, of course, are relative GCrich regions. A possible explanation is that $\mathrm{CMA}(+)$ regions in citrus are highly condensed repetitive DNA sites that are known as knobs in $Z$. mays (Chen et al., 2000). This idea is also supported by the present results in which 13 GISH signals were detected on the CMA $(+)$ regions and other GISH signals were detected on the condensed blocks of heterochromatin (Fig. 2A, D). 
In the present study, colorations of distinct GISH signals were red, yellow, and green (Figs. 2D and 4). For the GISH signals detected on the CMA $(+)$ regions, the colorations of one terminal, another terminal, and a proximal region in a type A chromosome were green, red, and yellow, respectively. In type $\mathrm{C}$ chromosome, a smaller green signal was detected in one terminal region and a medium red signal was detected in another terminal region. In the four chromosomes of the D2 type, three chromosomes revealed larger red signals, but one chromosome revealed a gradient pattern of yellow to red in one region. In the two chromosomes of the $\mathrm{D}_{1}$ type, one revealed a medium yellow signal and the other revealed a medium red signal. Both chromosomes of the $\mathrm{D}_{3}$ type revealed smaller red signals. On the other hand, five distinct GISH signals without CMA (+) regions were detected on the proximal region of two type $\mathrm{D}_{2}\left(\mathrm{D}_{2} \cdot \mathrm{Ry}\right.$ in Fig. 4$)$ and one type $\mathrm{D}_{3}$ chromosomes and on the terminal region of the one type $\mathrm{D}_{2}\left(\mathrm{D}_{2} \cdot \mathrm{RY}\right.$ in Fig. 4$)$ and the one type $\mathrm{E}_{\mathrm{M}}$ chromosome. Also, misty GISH signals were detected in type E chromosomes as well as in other types of chromosome without the region of distinct GISH signals. The size of the misty signal in $\mathrm{E}_{\mathrm{L}}$ chromosomes was the largest of the type $\mathrm{E}$ chromosomes. Of four $\mathrm{E}_{\mathrm{M}}$ chromosomes, the misty signals of two chromosomes were clearly larger than the other two chromosomes. The size of the misty signal in two $E_{S}$ chromosomes was small.

These results showed that the same types of chromosomes based on the relative sizes of $\mathrm{CMA}(+)$ regions and relative chromosome length by CMA and Giemsa staining were distinguishable to more detailed categories by GISH signals (Fig. 4). That is, two chromosomes of $\mathrm{D}_{1}$ type were classified to one chromosome with a yellow signal $\left(\mathrm{D}_{1} \cdot \mathrm{Y}\right)$ and one chromosome with a red signal $\left(D_{1} \cdot R\right)$; four chromosomes of $D_{2}$ type were classified to one chromosome with a red signal $\left(D_{2} \cdot R\right)$, two chromosomes with red and additional yellow signals $\left(\mathrm{D}_{2} \cdot \mathrm{Ry}\right)$, and one chromosome with a gradient signal of yellow to red $\left(D_{2} \cdot R Y\right)$; and four chromosomes of $E_{M}$ type were classified to two chromosomes with lager misty signals $\left(\mathrm{E}_{\mathrm{M}} \cdot \mathrm{Lm}\right)$ and two chromosomes with smaller ones $\left(\mathrm{E}_{\mathrm{M}} \cdot \mathrm{Sm}\right)$. The karyotype could be represented as $1 \mathrm{~A}+1 \mathrm{C}_{2}+1 \mathrm{D}_{1} \cdot \mathrm{Y}+$
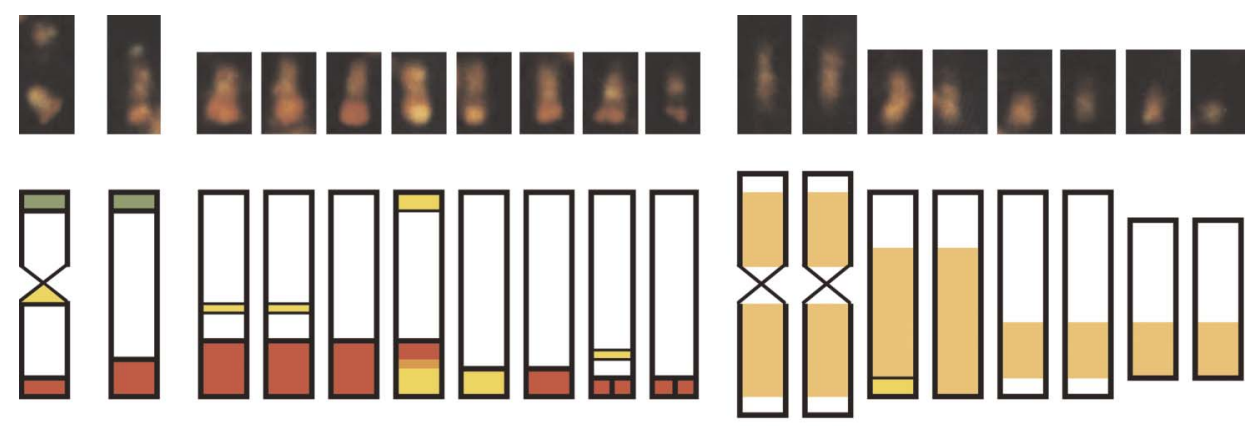

A

$\mathrm{C}_{2}$
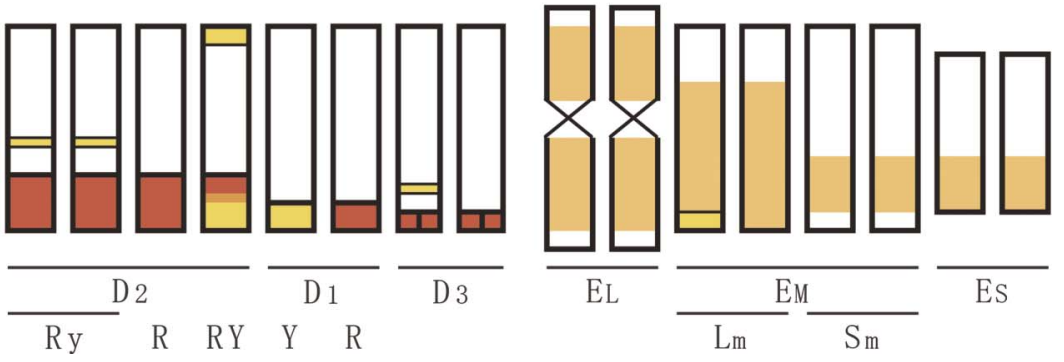

Fig. 4. Idiogram with picture images of satsuma mandarin chromosomes by genomic in situ hybridization (GISH). Double-probe DNAs from 'Yoshida' ponkan detected with red rhodamine and from 'Banpeiyu' pummelo detected with green fluorescein isothiocyanate were used for GISH. Chromosomes were grouped into 12 categories by an additional index of GISH signal coloration (see Fig. 2.), A, $\mathrm{C}_{2}, \mathrm{D}_{2} \cdot \mathrm{Ry}$ (red and yellow signals), $\mathrm{D}_{2} \cdot \mathrm{R}$ (red signal), $\mathrm{D}_{2} \cdot \mathrm{RY}$ [red with yellow signals on chromomycin $\mathrm{A}_{3}$ (CMA)-positive $(+)$ regions], $\mathrm{D}_{1} \cdot \mathrm{Y}$ (yellow signal), $\mathrm{D}_{1} \cdot \mathrm{R}, \mathrm{D}_{3}, \mathrm{E}_{\mathrm{L}}, \mathrm{E}_{\mathrm{M}} \cdot \mathrm{Lm}$ (long misty GISH signal), $\mathrm{E}_{\mathrm{M}} \cdot \mathrm{Sm}$ (short misty GISH signal), and $\mathrm{E}_{\mathrm{S}}$. The karyotype is $1 \mathrm{~A}+1 \mathrm{C}_{2}+1 \mathrm{D}_{1} \cdot \mathrm{Y}+1 \mathrm{D}_{1} \cdot \mathrm{R}+2 \mathrm{D}_{2} \cdot \mathrm{Ry}+1 \mathrm{D}_{2} \cdot \mathrm{R}+1 \mathrm{D}_{2} \cdot \mathrm{RY}+2 \mathrm{D}_{3}+2 \mathrm{E}_{\mathrm{L}}+2 \mathrm{E}_{\mathrm{M}} \cdot \mathrm{Lm}+2 \mathrm{E}_{\mathrm{M}} \cdot \mathrm{Sm}+2 \mathrm{E}_{\mathrm{S}}$ Six pairs of chromosomes would be homologous. Six monochromosome types were clearly distinguishable from each other. Each chromosome of satsuma mandarin could be identified by additional GISH.
$1 \mathrm{D}_{1} \cdot \mathrm{R}+2 \mathrm{D}_{2} \cdot \mathrm{Ry}+1 \mathrm{D}_{2} \cdot \mathrm{R}+1 \mathrm{D}_{2} \cdot \mathrm{RY}+2 \mathrm{D}_{3}+2 \mathrm{E}_{\mathrm{L}}+2 \mathrm{E}_{\mathrm{M}} \cdot \mathrm{Lm}+$ $2 \mathrm{E}_{\mathrm{M}} \cdot \mathrm{Sm}+2 \mathrm{E}_{\mathrm{S}}$. By the GISH method, 18 chromosomes of satsuma mandarin were categorized into 12 groups. It was indicated that six pairs of chromosomes, that is, two chromosomes of $\mathrm{D}_{2} \cdot \mathrm{Ry}, \mathrm{D}_{3}, \mathrm{E}_{\mathrm{L}}, \mathrm{E}_{\mathrm{M}} \cdot \mathrm{Lm}, \mathrm{E}_{\mathrm{M}} \cdot \mathrm{Sm}$, and $\mathrm{E}_{\mathrm{S}}$, would be homologous. Moreover, six chromosomes, types $\mathrm{A}, \mathrm{C}_{2}$ and $\mathrm{D}_{1} \cdot \mathrm{Y}, \mathrm{D}_{1} \cdot \mathrm{R}, \mathrm{D}_{2} \cdot \mathrm{R}$, and $\mathrm{D}_{2} \cdot \mathrm{RY}$, were clearly distinguishable om each other. Therefore, each chromosome of satsuma without CMA staining may be useful for chromosome only for chromosome analysis, but also for the construction of a cytological map. It has become possible in satsuma mandarin to confirm the correlation linkage group with chromosomes dependof chromosomes based on the relative sizes of CMA signals, could be detected by GISH. This new information is valuable for

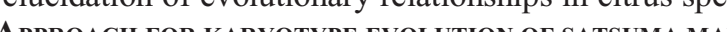
BY GENOMIC IN SITU HYBRIDIZATION SIGNALS. Citron ( $C$. medica L.), pummelo ( $C$. maxima), and one of the mandarins were theorized to be the original citrus species (Coletta Filho et al., 1998; Davies and Albrigo, 1994a; Handa et al., 1986; Nicolosi et al., 2000), whereas mandarins have been classified into many species (Tanaka, 1977). By analysis of morphological characterstics based on qualification theory, Handa and Oogaki (1985) group in the citrus species, have been divided into two or three (1) Satsuma mandarin has been thought to be born in around 1600 and Chinese mandarins such as honchiso (C. succosa hort. ex Tanaka), mankitsu (C. tardiferax hort. ex Tanaka), and sokitsu (C. subcompressa hort. ex Tanaka) (Japanese pronunciation) have been thought to be related to the seedling as its possible progenitor (Tanaka, 1948). Handa and Oogaki (1985) suggested that satsuma mandarin is a hybrid because it is more closely related to sweet orange [C. sinensis (L.) Osbeck] than mandarins by qualification theory analysis. Coletta Filho et al. (1998) demonstrated that mandarins were divided into two big groups and that satsuma mandarin belonged to a different group from ponkan (C. reticulata), which was a core mandarin by randomly amplified polymorphic DNA analysis. Federici et al. (1998) demonstrated that the ponkan group did not include satsuma mandarin, whereas it included 'Valencia' sweet orange by restricted fragment length 
polymorphism analysis. Moreover, Fang et al. (1998) demonstrated that both ponkan and satsuma mandarin were included in subgroup III, which was the biggest group in mandarin by intersimple sequence repeat marker analysis. Thus, phylogenic taxonomy in citrus is not easy by the conventional methods of DNA analysis as a result of conflicting evidence.

In citrus chromosome research, Befu et al. (2001) suggested that type D and type E chromosomes, which were observed in all the investigated citrus species, are original citrus chromosomes, and that A, B, and C type chromosomes were developed from the type D chromosome. Yamamoto and Tominaga (2003) investigated chromosomes in 17 species or cultivars of mandarins and identified the small numbers of types $\mathrm{A}, \mathrm{B}$, and $\mathrm{C}$ chromosomes. Cornelio et al. (2003) speculated that the species with the simplest karyotype (no A, B, or C chromosomes) and with a simple (no $\mathrm{A}$ and $\mathrm{B}$ chromosomes) and homozygous karyotype are the best candidates to represent a true species of mandarin. Moreover, the karyotype of citron is confirmed as 2B+8D+8E (Befu et al., 2001; Carvalho et al., 2005; Yamamoto et al., 2007), and the type A chromosome is included in all pummelos, whereas the type B chromosome is only present in some pummelos (Befu et al., 2000, 2001, 2002; Guerra, 1993; Miranda et al., 1997a). These results indicate that the type A chromosome originated from pummelo and the type B chromosome from citron. In addition, one of the papedas $(C$. micrantha Wester, C. macroptera Mont., and C. hystrix DC.) was supposed to be another original species in citrus. Yamamoto et al. (2007) reported that papedas have new types of chromosomes with different a CMA banding pattern from other citrus, that is, type $\mathrm{F}$ chromosome with one proximal band and type Dst chromosome with a satellite in type D. This result suggests that papedas are not direct ancestors of satsuma mandarin because it has no type F and Dst chromosomes.

The karyotypes of 'Banpeiyu' pummelo and 'Yoshida' ponkan, which were used as probe DNA in the present study, have already been reported by Befu et al. $(2001,2002)$ as $2 \mathrm{~A}+$ $2 \mathrm{~B}+2 \mathrm{C}+4 \mathrm{D}+8 \mathrm{E}$ and $1 \mathrm{~B}+1 \mathrm{C}+9 \mathrm{D}+7 \mathrm{E}$, respectively. In the present study, red GISH signals resulted from hybridization with the ponkan DNA probe and green GISH signals resulted from hybridization with the pummelo DNA probe. Red GISH signals were detected on nine chromosomes out of 10 on which GISH signals were detected on the CMA $(+)$ regions (Figs. 2D and 4 ). This result indicates that at least the red GISH signal regions originated from pure mandarin species and that six chromosomes of type D may be from mandarin. Moreover, the misty GISH signals, which were detected on the chromosome body except in the region of distinct GISH signals, were an almost orange color. From these results, satsuma mandarin must be very closely related to ponkan.

The GISH signals of type $\mathrm{C}$ chromosomes in satsuma mandarin were red on one terminal region and green on another. In the type A chromosome, moreover, red, green, and yellow signals were detected on one terminal, another terminal, and the proximal region, respectively. This is the first report that has found the existence of chromosomes with three different colored GISH signals in a fruit tree. This finding is also a rare case in plant chromosome research. Chromosomes with different GISH signals are very interesting and useful for studying their origin and history because the existence of types A and C chromosomes with both red and green signals means that both mandarin and pummelo are involved in the establishment of such chromosomes.
Considering the establishment of a chromosome with red and green signals, for example, in this type $\mathrm{C}$ chromosome, one individual of hybrid between mandarin and pummelo or a progeny, having both mandarin chromosomes with red signals and pummelo chromosomes with green signals, can produce a chromosome with both red and green signals by translocation at the meiosis phase. Therefore, satsuma mandarin is a progeny from a certain hybrid of individuals between mandarin and pummelo. Nicolosi et al. (2000) reported that the total number of DNA markers detected in satsuma mandarin was 49, two of which were sweet orange markers derived from pummelo, and the other 46 were common mandarin markers. This is supported by our result in which GISH signals derived from both mandarin and pummelo were detected on a chromosome of satsuma mandarin. Because the karyotype of sweet orange is $2 \mathrm{~B}+2 \mathrm{C}+7 \mathrm{D}+7 \mathrm{E}$ (Befu et al., 2000; Cornelio et al., 2003; Guerra, 1993; Miranda et al., 1997a; Yamamoto et al., 2007), perhaps the type $\mathrm{C}$ chromosome of satsuma mandarin is derived from sweet orange. Although the type A chromosome of satsuma mandarin cannot be directly derived from pummelo, at least part of the type A chromosome is originated from pummelo because both red and green signals were observed in the type A chromosome of satsuma mandarin. Therefore, it is considered that the type A chromosome of satsuma mandarin is derived from a certain mandarin with the type A chromosome such as honchiso [C. succosa (Chinese name = ben di zao)] (Miranda et al., 1997a; Yamamoto and Tominaga, 2003), king (C. nobilis Lour.), or kunenbo (C. nobilis var. kunep Tan.) (Yamamoto and Tominaga, 2003).

Yellow GISH signals were detected on the proximal region of the type A chromosomes and the terminal region of two type $\mathrm{D}$ chromosomes identical to the CMA $(+)$ regions and on the other region of four type $\mathrm{D}$ and one type $\mathrm{E}$ chromosomes (Figs. 2D and 4). This result indicates that the regions of yellow signals in satsuma mandarin are homologous to both mandarin and pummelo and that some may also be homologous to other citrus species. Chromosome regions, which are homologous among species, possibly contain highly conserved sites such as rDNA. The rDNA fluorescent in situ hybridization (FISH) signals in Meiwa kumquat (Fortunella crassifolia Swingle) chromosomes were detected on the proximal region of two type A chromosomes and on the terminal region of two type $\mathrm{C}$ chromosomes (Miranda et al., 1997b). Furthermore, the 45S rDNA signals of FISH in sweet orange chromosomes were detected on each proximal region of two type B chromosomes and on the terminal region of one type $\mathrm{D}$ chromosomes (Matsuyama et al., 1996; Pedrosa et al., 2000). These regions correspond to secondary constructions or the nucleolus organizing region (NOR). Matsuyama et al. (1996) observed that the 45S DNA region of type B chromosomes was always stretched at the prometaphase stage. Pedrosa et al. (2000) demonstrated that $45 \mathrm{~S}$ rDNA sites were located in the three $\mathrm{CMA}^{+} / \mathrm{DAPI}^{-}$ bands. In the present study, there is no region of $\mathrm{CMA}^{+} / \mathrm{DAPI}^{-}$ bands in satsuma mandarin chromosomes (Fig. 2B-C). However, the proximal region of type A chromosome was sometimes stretched (Figs. 2C and 3). Therefore, the proximal region of type A and the terminal region of type $\mathrm{D}$ chromosomes with yellow GISH signal in satsuma mandarin may be related to NOR.

In the present study, it was found that GISH is a powerful tool for the identification of individual and homologous chromosomes and karyotyping in citrus species. 


\section{Literature Cited}

Befu, M., A. Kitajima, and K. Hasegawa. 2001. Chromosome composition of some citrus species and cultivars based on chromomycin A3 (CMA) banding patterns [in Japanese with English summary]. J. Jpn. Soc. Hort. Sci. 70:83-88.

Befu, M., A. Kitajima, and K. Hasegawa. 2002. Classification of the citrus chromosomes with same types of chromomycin A banding patterns [in Japanese with English summary]. J. Jpn. Soc. Hort. Sci. 71:394-400.

Befu, M., A. Kitajima, X. Yang, and K. Hasegawa. 2000. Classification of 'Tosa-Buntan' pummelo (Citrus grandis [L.] Osb.) and 'Washington' navel orange [C. sinensis (L.) Osb.] and triforiate orange [Poncirus trifoliata (L.) Raf.] chromosome using young leaves. J. Jpn. Soc. Hort. Sci. 69:22-28.

Cai, Q., C.L. Guy, and G.A. Moore. 1994. Extension of the linkage map in Citrus using random amplified polymorphic DNA (RAPD) marker and RFLP mapping of cold-acclimation-responsive loci. Theor. Appl. Genet. 89:606-614.

Carvalho, R., W.S. Soares, A.C. Brasileoro-Vidal, and M. Guerra. 2005. The relationships among lemons, limes and citron: A chromosomal comparison. Cytogenet. Genome Res. 109:276-282.

Chen, C.C., C.M. Chen, F.C. Hsu, C.J. Wang, J.T. Yang, and Y.Y. Kao. 2000. The pachytene chromosomes of maize as revealed by fluorescence in situ hybridization with repetitive DNA sequences. Theor. Appl. Genet. 101:30-36.

Choi, Y., R. Tao, K. Yonemori, and A. Sugiura. 2002. Multi-color genomic in situ hybridization identifies parental chromosomes in somatic hybrids of Diospyros kaki and D. glandulosa. HortScience 37:184-186.

Coletta Filho, H.D., M.A. Machado, M.L.P.N. Targon, M.C.P.Q.D.G. Moreira, and J. Pompeu, Jr. 1998. Analysis of the genetic diversity among mandarins (Citrus spp.) using RAPD markers. Euphytica 102:133-139.

Cornelio, M.T.M.N., A.R.S. Figueiroa, K.G.B. Santos, R. Carvalho, W.S. Soares Filho, and M. Guerra. 2003. Chromosomal relationships among cultivars of Citrus reticulate Blanco, its hybrids and related species. Plant Syst. Evol. 240:149-161.

Davies, F.S. and L.G. Albrigo. 1994a. Taxonomy, cultivars and breeding, p. 12-23. In: F.S. Davies and L.G. Albrigo (eds.). Citrus. CAB International, Wallingford, UK.

Davies, F.S. and L.G. Albrigo. 1994b. Taxonomy, cultivars and breeding, p. 29. In: F.S. Davies and L.G. Albrigo (eds.). Citrus. CAB International, Wallingford, UK.

Fang, D.Q., R.R. Krueger, and M.L. Roose. 1998. Phylogenetic relationships among selected Citrus germplasm accessions revealed by inter-simple sequence repeat (ISSR) markers. J. Amer. Soc. Hort. Sci. 123:612-617.

Federici, C.T., D.Q. Fang, R.W. Scora, and M.L. Roose. 1998. Phylogenetic relationships within the genus Citrus (Rutaceae) and related genera as revealed by RFLP and RAPD analysis. Theol. Appl. Genet. 96:812-822.

Fu, C.H., C.L. Chen, W.W. Guo, and X.X. Deng. 2004. GISH, AFLP and PCR-RFLP analysis of an intergeneric hybrid combining Goutou sour orange and Poncirus triforiata. Plant Cell Rept. 23:391-396.

Garcia, R., M.J. Asins, J. Forner, and E. Carbonell. 1999. Genetic analysis of apomixis in Citrus and Poncirus by molecular markers. Theor. Appl. Genet. 99:511-518.

Guerra, M. 1993. Cytogenetics of Rutaceae. V. High chromosomal variability in Citrus species revealed by CMA/DAPI staining. Heredity 71:234-241.

Handa, T., Y. Iwamasa, and C. Oogaki. 1986. Phylogenic study of Fraction I protein in the genus Citrus and its close rerated genera. Jpn. J. Genet. 61:15-24.

Handa, T. and C. Oogaki. 1985. Numerical taxonomic study of Citrus L. and Fortunella Swingle using morphological charactersApplication of multivariate analysis [in Japanese with English summary]. J. Jpn. Soc. Hort. Sci. 54:145-154.
Kitajima, A., M. Befu, Y. Hidaka, T. Hotta, and K. Hasegawa. 2001. A chromosome preparation method using young leaves of citrus. J. Jpn. Soc. Hort. Sci. 70:191-194.

Maluszynska, J. and R. Hasterok. 2005. Identification of individual chromosomes and parental genomic in Brassica juncea using GISH and FISH. Cytogenet. Genome Res. 109:310-314.

Matsuyama, T., T. Akihama, Y. Ito, M. Omura, and K. Fukui. 1996. Characterization of heterochromatic regions in 'Trovita' orange (Citrus sinensis Osbeck) chromosomes by the fluorescent staining and FISH methods. Genome 39:941-945.

Miranda, M., F. Ikeda, T. Endo, T. Moriguchi, and M. Omura. 1997a. Comparative analysis on the distribution of heterochromatin in Citrus, Poncirus and Fortunella chromosomes. Chromosome Res. 5:86-92.

Miranda, M., F. Ikeda, T. Endo, T. Moriguchi, and M. Omura. 1997b. rDNA sites and heterochromatin in Meiwa kumquat (Fortunella crassifolia Swing.) chromosomes revealed by FISH and CMA/DAPI staining. Caryologia 50:333-340.

Nakano, M., H. Nesumi, T. Yoshioka, and T. Yoshida. 2001. Segregation of plants with undeveloped anthers among hybrids derived from the seed parent, 'Kiyomi' (Citrus unshiu $\times$ C. sinensis). J. Jpn. Soc. Hort. Sci. 70:539-545.

Nicolosi, E., Z.N. Deng, A. Gentile, S. La Malfa, G. Continella, and E. Tribulato. 2000. Citrus phylogeny and genetic origin of important species as investigated by molecular markers. Theor. Appl. Genet. 100:1155-1166.

Pedrosa, A., D. Schweizer, and M. Guerra. 2000. Cytological heterozygosity and the hybrid origin of sweet orange [Citrus sinensis (L.) Osbeck]. Theor. Appl. Genet. 100:361-367.

Poggio, L., G. Gonzalez, V. Confalonieri, C. Comas, and C.A. Naranjo. 2005. The genome organization and diversification of maize and its allied species revisited: Evidences from classical and FISH-GISH cytogenetic analysis. Cytogenet. Genome Res. 109:259-267.

Raina, S.N. and V. Rani. 2003. GISH technology in plant genome research. Methods Cell Sci. 23:83-104.

Ruiz, C. and M.J. Asins. 2003. Comparison between Poncirus and Citrus genetic linkage maps. Theor. Appl. Genet. 106:826-836.

Sankar, A.A. and G.A. Moore. 2001. Evaluation of inter-simple sequence repeat analysis for mapping in Citrus and extension of the genetic linkage map. Theor. Appl. Genet. 102:206-214.

Tanaka, T. 1977. Fundamental discussion of citrus classification. Studia Citrologica 14:1-6.

Tanaka, Y. 1948. Unshumikan, p. 428-432 [in Japanese]. In: Y. Tanaka (ed.). An iconograph of Japanese citrus fruits-A monographic study of species and varieties of citrus fruits grown in Japan. Vol. 2. Yokendo, Tokyo.

Ueno, I. and T. Shichijo. 1976. Parthenocarpy of tangor and tangelo using satsuma mandarin as seed parent [in Japanese]. J. Jpn. Soc. Hort. Sci. 46(suppl 1):94-95 (abstr.).

Yamaguchi, K., A. Kitajima, and K. Hasegawa. 2002. Availability of PI/DAPI staining for chromosome identification in citrus [in Japanese]. Chugoku-Shikoku Br. Jpn. Soc. Hort. Sci. 41:27 (abstr.).

Yamamoto, M., A.A. Abkenar, R. Matsumoto, H. Nesumi, T. Yoshida, T. Kuniga, T. Kubo, and S. Tominaga. 2007. CMA banding patterns of chromosome in major Citrus species. J. Jpn. Soc. Hort. Sci. 76: $36-40$.

Yamamoto, M., R. Matsumoto, and Y. Yamada. 1995. Relationship between sterility and seedlessness in citrus. J. Jpn. Soc. Hort. Sci. 64:23-29.

Yamamoto, M. and S. Tominaga. 2003. High chromosome variability of mandarins (Citrus spp.) revealed by CMA banding. Euphytica 129:267-274.

Yoshida, T. and Y. Mitsuoka. 1993. Difference in rate of citrus tristeza virus multiplication among citrus cultivars [in Japanese]. J. Jpn. Soc. Hort. Sci. 74(suppl 2):70-71 (abstr.).

Yoshimura, F., Y. Ono, T. Kawakita, and K. Matsuno. 1963. Studies on the cold injury of citrus trees [in Japanese]. J. Jpn. Soc. Hort. Sci. 32:149-156. 